\title{
Induction of DT-Diaphorase in Riboflavin-Deficient Rats by Xenobiotic Treatment
}

\author{
Kunio YAGI, ${ }^{1, *}$ Jin-Yang GuAN, ${ }^{1, \dagger}$ Talia MIron, ${ }^{1}$ \\ Sadaaki Komura, ${ }^{1}$ Nobuko OHISHI, ${ }^{1}$ and Lars ERNSTER ${ }^{2}$ \\ ${ }^{1}$ Institute of Applied Biochemistry, Yagi Memorial Park, Mitake, Gifu 505-01, Japan \\ ${ }^{2}$ Department of Biochemistry, Arrhenius Laboratory for Natural Sciences, \\ Stockholm University, S-10691 Stockholm, Sweden
}

(Received July 1, 1996)

Summary This paper describes the induction of DT-diaphorase by xenobiotic treatment of riboflavin-deficient rats. Upon injection of $\beta$ naphthoflavone once a day for 3 successive days into male Wistar rats fed a riboflavin-supplemented diet for 4 weeks, the activity of DT-diaphorase in the cytosolic, mitochondrial, and microsomal fractions of rat liver increased several fold compared with that of the vehicle control. In the case of rats fed a riboflavin-deficient diet for 4 weeks, DT-diaphorase activity in the cytosolic fraction was markedly decreased and that in microsomes was somewhat diminished, whereas the activity in mitochondrial fraction did not change, compared with that of the above control. However, when the deficient rats were injected with $\beta$-naphthoflavone intraperitoneally, DT-diaphorase activity in the cytosolic, mitochondrial, and microsomal fractions increased in spite of a significant decrease in flavin levels in the body. These results indicate that DT-diaphorase is actually induced even under conditions of riboflavin deficiency and that the apoprotein of the enzyme takes up FAD to become holoenzyme, suggesting a redistribution of FAD among various flavoproteins in the liver of riboflavin-deficient rats upon administration of a xenobiotic. Among the activities of other flavoenzymes examined, the occurrence of the apoenzyme of glutathione reductase, thioredoxin reductase or NADPH-cytochrome P450 reductase was found upon the injection of $\beta$-naphthoflavone in the riboflavin-deficient rats, which might be an indication of the above-mentioned redistribution.

Key Words: DT-diaphorase, riboflavin deficiency, xenobiotic, $\beta$-naphthoflavone

\footnotetext{
* To whom correspondence should be addressed.

† Present address: Institute of Basic Medicine of Liaoning Province, Shenyang, China.
} 
It is well recognized that lipid peroxides and their related free radicals are involved in the pathogenesis of various diseases and aging [1]. Lipid peroxidation is provoked by many causes. Previously we reported that riboflavin deficiency in rats resulted in an increase in the lipid peroxide level in the serum [2]. An increase in the lipid peroxide level was also found in the liver microsomes of riboflavindeficient rats [3]. In these cases, the activity of glutathione reductase, which requires FAD as a prosthetic group, was found to be decreased $[2,3]$. Such a decrease in the enzyme activity brought about a decrease in the level of reduced glutathione, and this in turn diminished the capability of the lipid hydroperoxidedegrading enzyme systems.

Another flavoprotein, DT-diaphorase (EC 1.6.99.2), catalyzes the two-electron reduction of quinones to hydroquinones, preventing the formation of superoxide anions through redox cycling of semiquinones via one-electron quinone reductases [4]. Thus, DT-diaphorase is also considered to act as an antioxidative enzyme. We have previously reported that the activity of DT-diaphorase in the cytosolic fraction of the liver from rats fed a riboflavin-deficient diet is significantly decreased as compared with that of animals fed a riboflavin-supplemented diet [5]. In that report, we demonstrated that the decrease in the enzyme activity was accompanied by a decrease in the amount of the protein of this enzyme. Taking into account the role of DT-diaphorase in preventing superoxide formation, we considered that the decrease in DT-diaphorase activity also could be one of the causes of the elevation of the lipid peroxide level observed in riboflavin-deficient rats.

It is well known that DT-diaphorase is inducible by a wide variety of compounds such as 3 -methylcholanthrene, $\beta$-naphthoflavone, and $t$-butylhydroquinone [6-8]. As to the molecular mechanisms underlying the expression of the gene of this enzyme, two responsive elements have been identified in the 5 -flanking region of the rat gene $[9,10]$. One of these is referred to as the "xenobiotic responsive element," and the other as the "antioxidant responsive element." Hence, the question arose as to whether DT-diaphorase is inducible in riboflavin-deficient rats. The present paper demonstrates that induction of DT-diaphorase actually occurs in these rats upon treatment with a xenobiotic.

\section{MATERIALS AND METHODS}

Male rats of the Wistar strain, weighing about $40 \mathrm{~g}$, were purchased from SLC, Co., Ltd., Hamamatsu, Japan. The basal riboflavin-deficient diet was prepared as described in a previous paper [2]. It was composed of vitamin-free casein (24.5\%), corn starch $(45.5 \%)$, sucrose (10\%), corn oil (6\%), cellulose (5\%), $\alpha$-starch (1\%), vitamin mixture $(1 \%)$, and salt mixture $(7 \%)$. The content of riboflavin in the diet was examined and found to be below $12 \mu \mathrm{g}$ per $100 \mathrm{~g}$ of the diet. The riboflavinsupplemented diet was the above-mentioned basal riboflavin-deficient diet supplemented with $1.4 \mathrm{mg}$ of riboflavin per $100 \mathrm{~g}$ diet. 
The animals were divided into four groups. Rats of groups A and B were given the riboflavin-supplemented diet, and animals of groups $\mathrm{C}$ and $\mathrm{D}$, the riboflavin-deficient diet, for 4 weeks. The intake of the diet was controlled to be the same among the four groups during the period of feeding. The animals were given water ad libitum. After 4 weeks, the rats of groups B and D were given $\beta$-naphthoflavone in corn oil intraperitoneally at a dose of $20 \mathrm{mg} / \mathrm{kg}$ body weight once a day for three successive days; and the animals of groups $\mathrm{A}$ and $\mathrm{C}$, only corn oil. One day after the last injection of the xenobiotic or vehicle, the animals were sacrificed under anesthesia with diethyl ether. Their livers were excised, perfused with chilled physiological saline, and homogenized in 4 volumes of $10 \mathrm{~mm}$ Tris- $\mathrm{HCl}$ buffer $(\mathrm{pH} 7.5)$ containing $0.25 \mathrm{M}$ sucrose; and then mitochondrial, microsomal, and cytosolic fractions were obtained by differential centrifugation according to the method of Hogeboom [11].

DT-diaphorase activity in the cytosolic fraction was measured by the method of Ernster [12] and that in the mitochondrial and microsomal fractions, by the method of Lind and Höjeberg [13]. Assay for cytosolic glutathione reductase and thioredoxin reductase activities was carried out according to Nichoalds [14] and Luthman and Holmgren [15], respectively. Microsomal NADPH-cytochrome P450 reductase activity was measured according to Strobel and Dignam [16] and NADH-cytochrome $b_{5}$ reductase activity, according to Mihara and Sato [17]. Succinate dehydrogenase activity was measured as described by Ackrell et al. [18]. D-Amino acid oxidase activity was measured as described [19]. One unit of enzyme activity was defined as the amount of the enzyme that oxidizes or reduces $1 \mu \mathrm{mol}$ of substrate per min.

\section{RESULTS AND DISCUSSION}

\section{Body weight gain}

The body weights (mean \pm SD) of rats in groups A, B, C, and D were $74.0 \pm$ $3.0,73.4 \pm 1.3,62.4 \pm 7.3$, and $70.8 \pm 10.3 \mathrm{~g}$, respectively. The liver weights of animals in the four groups were $3.1 \pm 0.1,4.1 \pm 0.3,2.3 \pm 0.5$, and $4.2 \pm 0.6 \mathrm{~g}$, respectively. This indicates that the liver weight of rats given the xenobiotic $\beta$-naphthoflavone was higher than that of the corresponding animals without the xenobiotic.

\section{Effect of $\beta$-naphthoflavone on DT-diaphorase activity in the cytosolic fraction}

DT-diaphorase activity in the cytosolic fractions of the four groups of rats is shown in Fig. 1. The enzyme activity of rats fed the riboflavin-supplemented diet (group A) was about 1 unit/mg protein. When animals were fed the riboflavin-deficient diet for 4 weeks (group C), the activity of the enzyme was markedly decreased. Upon injection of $\beta$-naphthoflavone into rats fed the riboflavinsupplemented diet (group B), the activity of DT-diaphorase increased about 6 fold over that of group A animals. Such an effect of the xenobiotic was also the case for animals under riboflavin deficiency. Thus, the activity in the group D animals 
was significantly higher than that in the group $\mathrm{C}$ animals, even though the level of the enzyme activity in the group D animals was less than that of group B animals.

Figure 1 also shows the changes in DT-diaphorase activity determined with addition of FAD to the assay mixture. As seen in the figure, DT-diaphorase activity was not changed by the addition of FAD in any of the groups. This indicates that DT-diaphorase exists in the form of holoenzyme even under the conditions of riboflavin deficiency and/or $\beta$-naphthoflavone injection. This finding is consistent with the concept that the increase in the activity caused by the injection of $\beta$-naphthoflavone is due to an increase in the amount of enzyme protein.

Western blot analysis of DT-diaphorase in the cytosolic fraction

Figure 2 shows the amount of DT-diaphorase protein in the cytosolic fraction of rat liver. As can be seen, the amount of protein that was immunoreactive with the anti-DT-diaphorase antibody decreased in animals fed the riboflavin-deficient diet (group C) as compared with that in animals fed the riboflavin-supplemented diet (group A). When animals were injected with $\beta$-naphthoflavone (groups B and D), the amount of the immunoreactive protein increased in both of them compared with the amount in the corresponding vehicle controls.

\section{Effect of $\beta$-naphthoflavone on DT-diaphorase activity in the mitochondrial fraction}

Figure 3 shows DT-diaphorase activity in the mitochondrial fraction of rat liver. As seen in the figure, no significant difference was observed in the activity between rats fed the riboflavin-supplemented and deficient diet (group A vs. group C). Upon the injection of $\beta$-naphthoflavone, mitochondrial DT-diaphorase activ-

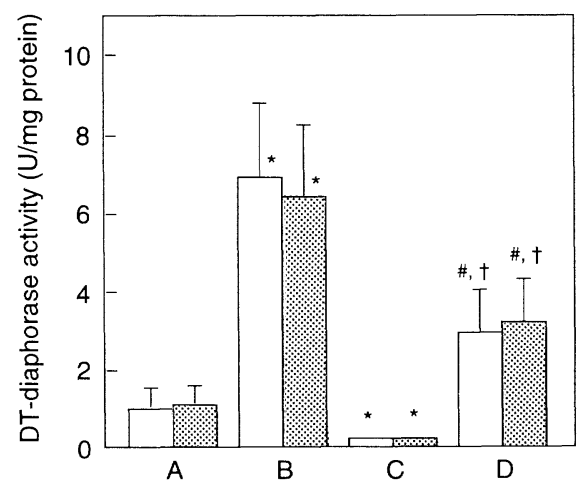

Fig. 1. Effect of $\beta$-naphthoflavone on DT-diaphorase activity in rat liver cytosol. The activity was determined in the absence $(\square)$ or presence (장 of FAD. Mean \pm SD is given. $n=5$. Significant difference, ${ }^{*} p<0.01$ from group $\mathrm{A} ;{ }^{\sharp} p<0.01$ from group $\mathrm{B} ;{ }^{\dagger} p<0.01$ from group C. Groups A, B, C, and D indicate each experimental group as defined in "Materials and Methods." 
ity was increased in both groups B and D, as in the case of cytosolic DTdiaphorase.

\section{Effect of $\beta$-naphthoflavone on DT-diaphorase activity in the microsomal fraction}

The microsomal DT-diaphorase activity was also examined. Figure 4 shows that the activity in the liver microsomal fraction of rats fed the riboflavin-deficient diet (group C) was somewhat diminished as compared with that of animals fed riboflavin-supplemented diet (group A). Similar to the cytosolic enzyme, the activity markedly increased after the injection of $\beta$-naphthoflavone in animals fed either the riboflavin-supplemented or riboflavin-deficient diet (groups B and D).

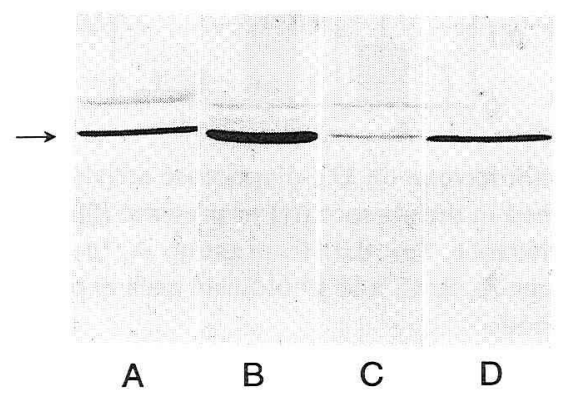

Fig. 2. Western blot analysis of DT-diaphorase. Rat liver cytosolic proteins ( $35 \mu \mathrm{g})$ in each group were subjected to sodium dodecyl sulfate-polyacrylamide gel electrophoresis. Groups A, B, C, and D were defined in "Materials and Methods." Arrow indicates DT-diaphorase (ca. $30 \mathrm{kDa})$.

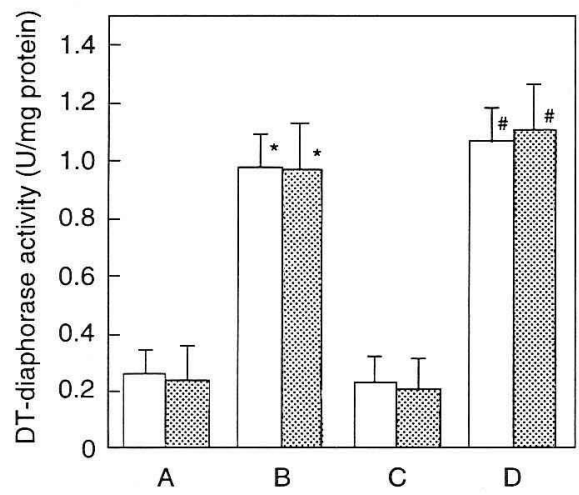

Fig. 3. Effect of $\beta$-naphthoflavone on DT-diaphorase activity in rat liver mitochondria. The activity was determined in the absence ( $\square$ ) or presence (圆) of FAD. Mean \pm SD is given. $n=5$. Significant difference, ${ }^{*} p<0.01$ from group $\mathrm{A} ;{ }^{*} p<0.01$ from group C. Groups A, B, C, and D indicate each experimental group as defined in "Materials and Methods." 


\section{Effect of $\beta$-naphthoflavone on the activities of various other flavoenzymes}

Table 1 summarizes changes in the activities of various other flavoenzymes in the cytosolic, mitochondrial, and microsomal fractions after the injection of $\beta$ -

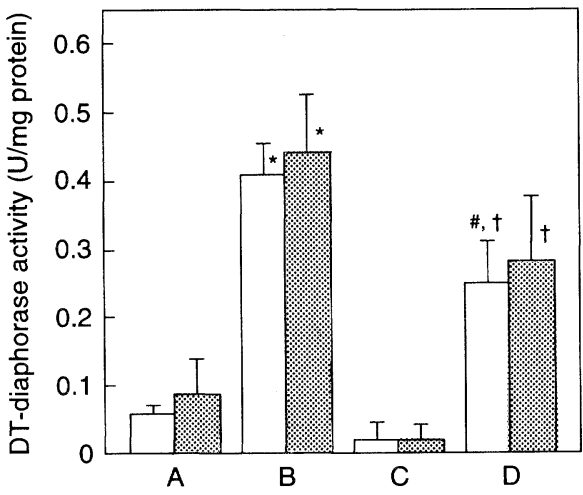

Fig. 4. Effect of $\beta$-naphthoflavone on DT-diaphorase activity in rat liver microsomes. The activity was determined in the absence $(\square)$ or presence (장) of FAD. Mean \pm SD is given. $n=3$. Significant difference, ${ }^{*} p<0.01$ from group $\mathrm{A} ;{ }^{\sharp} p<0.01$ from group $\mathrm{B} ;{ }^{\dagger} p<0.01$ from group C. Groups A, B, C, and D indicate each experimental group as defined in "Materials and Methods."

Table 1. Effect of $\beta$-naphthoflavone on the activities of various flavoenzymes in rat liver.

\begin{tabular}{|c|c|c|c|c|}
\hline \multirow{2}{*}{ Flavoenzymes } & \multicolumn{4}{|c|}{ Groups } \\
\hline & A & $\mathrm{B}$ & $\mathrm{C}$ & $\mathrm{D}$ \\
\hline \multicolumn{5}{|l|}{ Cytosolic fraction } \\
\hline GR $(-F A D)$ & $125 \pm 10$ & $160 \pm 8^{* *}$ & $61 \pm 14^{* *}$ & $66 \pm 8$ \#\# \\
\hline$(+\mathrm{FAD})$ & $123 \pm 11$ & $151 \pm 12^{*}$ & $81 \pm 25^{*}$ & $92 \pm 8^{\#,+\#}$ \\
\hline TR $(-F A D)$ & $7.5 \pm 1.1$ & $8.0 \pm 1.8$ & $2.9 \pm 0.7^{* *}$ & $3.4 \pm 0.5^{\#}$ \\
\hline$(+\mathrm{FAD})$ & $9.3 \pm 2.6$ & $9.2 \pm 1.3$ & $4.1 \pm 0.2^{*, \ddagger}$ & $6.0 \pm 1.5^{\#, \neq}$ \\
\hline \multicolumn{5}{|c|}{ Mitochondrial and peroxisomal fraction } \\
\hline SDH $(-$ FAD $)$ & $420 \pm 39$ & $412 \pm 28$ & $238 \pm 90^{*}$ & $205 \pm 29 \# \#$ \\
\hline$(+\mathrm{FAD})$ & $433 \pm 44$ & $411 \pm 27$ & $231 \pm 92 *$ & $204 \pm 28 \#$ \\
\hline $\mathrm{DAO}(-\mathrm{FAD})$ & $1.00 \pm 0.18$ & $1.21 \pm 0.74$ & nd & nd \\
\hline$(+\mathrm{FAD})$ & $2.45 \pm 0.27$ 柿 & $2.64 \pm 0.11^{\ddagger}$ & nd & nd \\
\hline \multicolumn{5}{|l|}{ Microsomal fraction } \\
\hline P450 Red $(-$ FAD, - FMN $)$ & $185 \pm 9$ & $179 \pm 31$ & $64 \pm 14^{* *}$ & $31 \pm 10^{\#, \dagger}$ \\
\hline$(+\mathrm{FAD},+\mathrm{FMN})$ & $188 \pm 43$ & $208 \pm 14$ & $121 \pm 25^{*,+\ddagger}$ & $91 \pm 11$ \#, 杉 \\
\hline$b_{5} \operatorname{Red}(-\mathrm{FAD})$ & $3.92 \pm 0.65$ & $4.09 \pm 0.33$ & $3.44 \pm 0.42$ & $3.36 \pm 0.25^{\#}$ \\
\hline$(+\mathrm{FAD})$ & $4.35 \pm 0.32$ & $4.57 \pm 0.32$ & $3.69 \pm 0.85$ & $3.60 \pm 0.36^{\#}$ \\
\hline
\end{tabular}

Enzyme activities are expressed as milliunit/mg protein. Mean value \pm SD is given. $n=3-5$. Abbreviations used are the following: GR, glutathione reductase; TR, thioredoxin reductase; $\mathrm{SDH}$, succinate dehydrogenase; DAO, D-amino acid oxidase; P450 Red, NADPH-cytochrome P450 reductase; $b_{5}$ Red, NADH-cytochrome $b_{5}$ reductase; nd, not detectable. Significant difference, ${ }^{*} p<0.05,{ }^{* *} p<0.01$ vs. group $\mathrm{A} ;{ }^{\sharp} p<0.05$, ${ }^{\#} p<0.01$ vs. group $\mathrm{B}$; and ${ }^{\dagger} p<$ 0.01 vs. group $\mathrm{C} .{ }^{\ddagger} p<0.05,{ }^{\star} p<0.01$ vs. activity determined without addition of $5 \mu \mathrm{M}$ flavin. 
naphthoflavone. In the case of the cytosolic fraction, the two flavoenzyme activities examined, glutathione reductase and thioredoxin reductase, were decreased in riboflavin-deficient animals. Injection of $\beta$-naphthoflavone into rats under the condition of riboflavin deficiency did not have any influence on these enzyme activities. Addition of FAD had no significant effect on the glutathione reductase in the riboflavin-deficient rats, but stimulated the activity in the animals given $\beta$-naphthoflavone. Thioredoxin reductase activity was stimulated in the C and D group animals when determined in the presence of exogenous FAD.

The activities of succinate dehydrogenase and D-amino acid oxidase in the mitochondrial-peroxisomal fraction were decreased under riboflavin deficiency; especially the latter enzyme activity decreased to almost nil. Injection of $\beta$-naphthoflavone had no effect on these mitochondrial and peroxisomal flavoenzyme activities. Addition of FAD had no effect on the succinate dehydrogenase, but stimulated the D-amino acid oxidase activity in the A and B group animals.

The activity of NADPH-cytochrome P450 reductase was markedly decreased by riboflavin deficiency. The level of this enzyme activity was further decreased by the injection of $\beta$-naphthoflavone into riboflavin-deficient rats when assayed in the absence of both FAD and FMN. When the enzyme activity was measured in the presence of added FAD and FMN, it was found to be significantly higher than that measured without addition of FAD and FMN in both groups $\mathrm{C}$ and $\mathrm{D}$. This indicates an accumulation of apoprotein of the enzyme under the conditions of riboflavin deficiency. The activity in animals fed the riboflavin-supplemented diet was not affected by treatment with $\beta$-naphthoflavone, as already reported by Shephard et al. [20].

The activity of NADH-cytochrome $b_{5}$ reductase was unaffected by riboflavin deficiency. This result is consistent with that reported previously [21]. We also found that the activity was unaffected by $\beta$-naphthoflavone treatment.

The present data clearly show that DT-diaphorase is induced by the injection of $\beta$-naphthoflavone in riboflavin-deficient rats and that the enzyme is present as a holoenzyme in spite of a remarkable decrease in flavin level. In fact, we measured the level of flavins in their liver and found that after feeding the rats with the riboflavin-deficient diet for 4 weeks, the level of flavins decreased to about $30 \%$ of that in the liver of animals fed the riboflavin-supplemented diet.

These data indicate that the apoprotein of DT-diaphorase produced in response to the xenobiotic injection takes up a sufficient amount of FAD to become the active holoenzyme. Since all FAD molecules are considered to be present as a form bound to proteins, that is, no free FAD exists in cells, the fact that DTdiaphorase induced by $\beta$-naphthoflavone treatment is the holoenzyme suggests the occurrence of a redistribution of FAD among flavoproteins. If this is the case, it can be expected that some flavoenzyme(s) should occur as the apoprotein upon xenobiotic treatment under the condition of riboflavin deficiency. When we examined possible changes in various flavoenzyme activities, we found that glutathione reductase and thioredoxin reductase occurred as the apoproteins after 
$\beta$-naphthoflavone treatment of riboflavin-deficient rats as judged by the FAD addition test. In addition, it is to be noted that the holoenzyme level of NADPHcytochrome $\mathrm{P} 450$ reductase was decreased, and a considerable amount of its apoprotein occurred upon xenobiotic treatment during riboflavin deficiency. These results strongly suggest the redistribution of FAD upon exposure of animals to the xenobiotics under riboflavin deficiency.

\section{REFERENCES}

1. Yagi, K. (1987): Lipid peroxides and human diseases. Chem. Phys. Lipids, 45, 337-351.

2. Yagi, K., Komura, S., Yoshino, K., Konishi, H., and Abe, H. (1989): Serum lipid peroxides and cataractogenesis in riboflavin deficiency. J. Clin. Biochem. Nutr., 6, 39-48.

3. Taniguchi, M., and Hara, T. (1983): Effects of riboflavin and selenium deficiencies on glutathione and its relating enzyme activities with respect to lipid peroxide content of rat livers. J. Nutr. Sci. Vitaminol., 29, 283-292.

4. Lind, C., Cadenas, E., Hochstein, P., and Ernster, L. (1990): DT-diaphorase: Purification, properties, and function. Methods Enzymol., 186, 287-301.

5. Yagi, K., Komura, S., Nakashima, M., Ishida, N., Ohishi, N., and Ernster, L. (1994): Effect of riboflavin deficiency on DT-diaphorase, in Flavins and Flavoproteins, ed. by Yagi, K., Walter de Gruyter \& Co., Berlin/New York, pp. 479-482.

6. Kumaki, K., Jensen, N.M., Shire, J.G.M., and Nebert, D.W. (1977): Genetic differences in induction of cytosol reduced-NAD(P):menadione oxidoreductase and microsomal aryl hydrocarbon hydroxylase in the mouse. J. Biol. Chem., 252, 157-165.

7. Williams, J.B., Wang, R., Lu, A.Y.H., and Pickett, C.B. (1984): Rat liver DT-diaphorase: Regulation of functional mRNA levels by 3-methylcholanthrene, trans-stilbene oxide, and phenobarbital. Arch. Biochem. Biophys., 232, 408-413.

8. De Long, M.J., Prochaska, H.J., and Talalay, P. (1986): Induction of NAD(P)H:quinone reductase in murine hepatoma cells by phenolic antioxidants, azo dyes, and chemoprotectors: A model system for the study of anticarcinogenicity. Proc. Natl. Acad. Sci., U.S.A., 83, 787-791.

9. Favreau, L.V., and Pickett, C.B. (1991): Transcriptional regulation of the rat NAD(P)H: quinone reductase gene. Identification of regulatory elements controlling basal level expression and inducible expression by planar aromatic compounds and phenolic antioxidants. $J$. Biol. Chem., 266, 4556-4561.

10. Favreau, L.V., and Pickett, C.B. (1995): The rat quinone reductase antioxidant response element. Identification of the nucleotide sequence for basal and inducible activity and detection of antioxidant response element-binding proteins in hepatoma and non-hepatoma cell lines. J. Biol. Chem., 270, 24468-24474.

11. Hogeboom, G.H. (1955): Fractionation of cell components of animal tissues. Methods Enzymol., 1, 16-19.

12. Ernster, L. (1967): DT-diaphorase. Methods Enzymol., 10, 309-317.

13. Lind, C., and Höjeberg, B. (1981): Biospecific adsorption of hepatic DT-diaphorase on immobilized dicoumarol. Arch. Biochem. Biophys., 207, 217-224.

14. Nichoalds, G.E. (1974): Assessment of status of riboflavin nutriture by assay of erythrocyte glutathione reductase activity. Clin. Chem., 20, 624-628.

15. Luthman, M., and Holmgren, A. (1982): Rat liver thioredoxin and thioredoxin reductase: Purification and characterization. Biochemistry, 21, 6628-6633.

16. Strobel, H.W., and Dignam, T.D. (1978): Purification and properties of NADPH-cytochrome P-450 reductase. Methods Enzymol., 52, 89-96.

17. Mihara, K., and Sato, R. (1972): Partial purification of NADH-cytochrome $b_{5}$ reductase from rabbit liver microsomes with detergents and its properties. J. Biochem., 71, 725-735.

J. Clin. Biochem. Nutr. 
18. Ackrell, B.A.C., Kearney, E.B., and Singer, T.P. (1978): Mammalian succinate dehydrogenase. Methods Enzymol., 53, 466-483.

19. Friedemann, T.E. (1957): Determination of $\alpha$-keto acids. Methods Enzymol., 3, 414-418.

20. Shephard, E.A., Phillips, I.R., Pike, S.F., Ashworth, A., and Rabin, B.R. (1982): Differential effect of phenobarbital and $\beta$-naphthoflavone on the mRNAs coding for cytochrome P450 and NADPH cytochrome P450 reductase. FEBS Lett., 150, 375-380.

21. Olpin, S.E., and Bates, C.J. (1982): Lipid metabolism in riboflavin-deficient rats. 2. Mitochondrial fatty acid oxidation and the microsomal desaturation pathway. Br. J. Nutr., 47, 589-596. 